\title{
Classification of an Agrosilvopastoral System Using RGB Imagery from an Unmanned Aerial Vehicle
}

\author{
Luís Pádua $^{1,2(\bowtie)}\left(\mathbb{D}\right.$, Nathalie Guimarães ${ }^{1}$, Telmo Adão ${ }^{1,2}$ (D), \\ Pedro Marques $^{1}$ (D), Emanuel Peres ${ }^{1,2}$ (D), António Sousa ${ }^{1,2}$ (D), \\ and Joaquim J. Sousa ${ }^{1,2}$ (D) \\ 1 School of Science and Technology, \\ University of Trás-os-Montes e Alto Douro, 5000-801 Vila Real, Portugal \\ \{luispadua, nsguimaraes, telmoadao, pedro.marques, \\ eperes, amrs, jjsousa\}@utad.pt \\ ${ }^{2}$ Centre for Robotics in Industry and Intelligent Systems (CRIIS), INESC \\ Technology and Science (INESC-TEC), Porto 4200-465, Portugal
}

\begin{abstract}
This paper explores the usage of unmanned aerial vehicles (UAVs) to acquire remotely sensed very high-resolution imagery for classification of an agrosilvopastoral system in a rural region of Portugal. Aerial data was obtained using a low-cost UAV, equipped with an RGB sensor. Acquired imagery undergone a photogrammetric processing pipeline to obtain different data products: an orthophoto mosaic, a canopy height model (CHM) and vegetation indices (VIs). A superpixel algorithm was then applied to the orthophoto mosaic, dividing the images into different objects. From each object, different features were obtained based in its maximum, mean, minimum and standard deviation. These features were extracted from the different data products: CHM, VIs, and color bands. Classification process - using random forest algorithm classified objects into five different classes: trees, low vegetation, shrubland, bare soil and infrastructures. Feature importance obtained from the training model showed that CHM-driven features have more importance when comparing to those obtained from VIs or color bands. An overall classification accuracy of $86.4 \%$ was obtained.
\end{abstract}

Keywords: Agrosilvopastoral systems - Unmanned aerial vehicles · Photogrammetric processing $\cdot$ Superpixels $\cdot$ Random forest

\section{Introduction}

Employing unmanned aerial vehicles (UAVs) in environmental monitoring activities is increasing, with a wide range of applications in forestry [1], agriculture [2], and grassland monitoring [3]. This remote sensing platform provides high flexibility by enabling superior temporal and spatial resolutions [4] with lower costs, in medium and small projects, when compared to traditional remote sensing platforms [5].

In the classification process of data, collected by UAVs, it is essential to select the best machine learning algorithm. There are three common algorithms being used in 
remote sensing: (1) random forest; (2) support vector machines; and (3) artificial neural networks. The selection of the most appropriate method is dependent on the type of problem being solved. In case of having multiple features but limited records, support vector machines might work better. In case of having a considerable number of records but less features, Neural Networks present better prediction/classification accuracy [6]. On the other hand, Random forest is a type of ensemble classifier that produces multiple decision trees by using a random subset of training samples and variables. It can handle high data dimensionality and multicollinearity swiftly and is insensitive to overfitting [7]. Indeed, random forest is widely used in remote sensing applications, being applied to urban vegetation mapping [8], land use and land cover mapping [9, 10], grassland classification [11] and tree species classification [12-14].

This study aims to classify an agrosilvopastoral system using a random forest algorithm and data driven from photogrammetric processing of UAV-based RGB imagery. Agrosilvopastoral systems can be defined as a collective name for land-use systems, which include the woody component (trees and/or shrubs), crops and cattle. Essentially, these systems are associated to a model of production and conservation centered on silvicultural practices and agricultural activities [15]. According to Nair [16] agrosilvopastoral systems "include the use of woody hedgerows for browse, mulch and green manure as well as for soil conservation".

This paper is structured as follows: the next section presents the study area, describes the UAV used and related imagery acquisition process. Methods used for imagery segmentation, features extracted, and the classification algorithm are also presented; Sect. 3 presents and discusses obtained results. The last section has some conclusions and describes steps towards future developments.

\section{Materials and Methods}

\subsection{Study Area}

The study area is located in north-eastern Portugal $\left(41^{\circ} 22^{\prime} 43.8^{\prime \prime} \mathrm{N}, 7^{\circ} 35^{\prime} 00.8^{\prime \prime} \mathrm{W}\right)$ and is composed of trees (mainly Pinus Pinaster and Castanea Sativa Mill.), shrubland communities (Cytisus striatus), low-land vegetation and crops, such as grassland and potatoes, along with bare soil areas and some men-made infrastructures. An area overview is presented in Fig. 1. Pine trees are present in both the north and east sections of the study area, with some small trees located in the central section. Two chestnut plantations are also present: one located in the central section and a smaller plantation in the north section. Regarding shrubland communities, those are located throughout the study area, being prevalent around its boarders. Low-land vegetation is mainly located in the south section and is composed of grassland and a potato plantation. Bare soil areas are spread throughout the study area, some covered by dry vegetation - mostly in between pine trees and shrubs - and some granite stones near man-made infrastructures. As for the latter, they represent a smaller area in the south and southwest sections of the study area and are used mainly as livestock accommodations. 


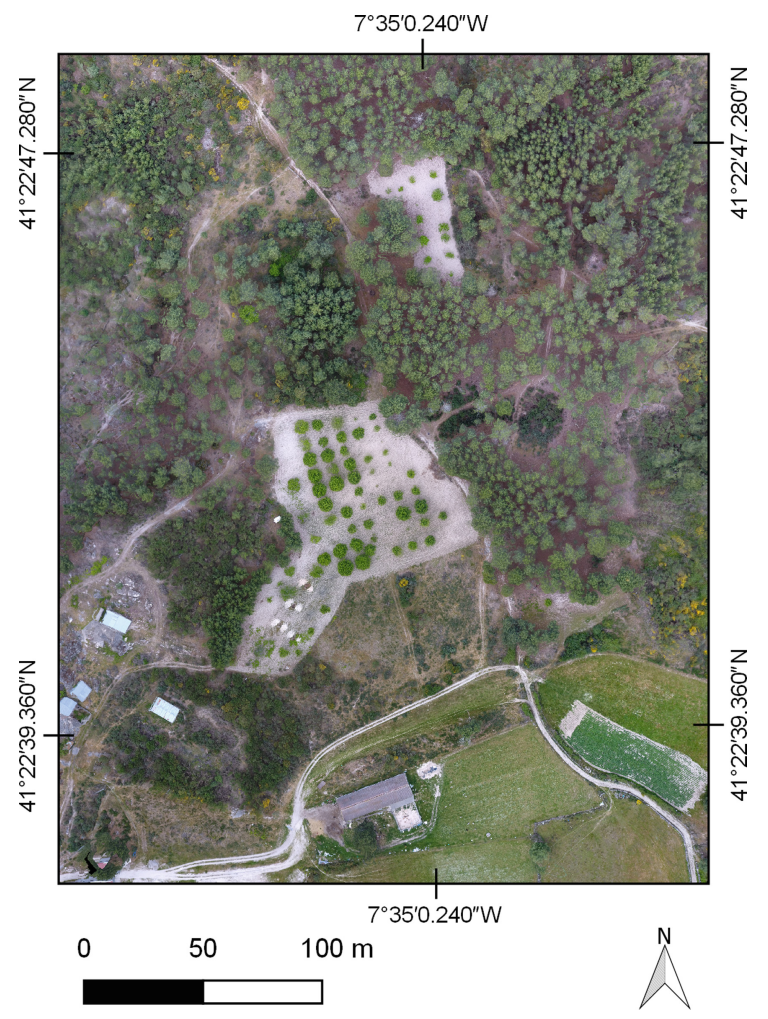

Fig. 1. General overview of the study area. Coordinates in WGS84 (EPSG:4326).

\subsection{Data Acquisition}

Aerial data acquisition was made using the DJI Phantom 4 (DJI, Shenzhen, China). It is cost-effective multi-rotor UAV equipped with a Global Navigation Satellite System (GNSS) receiver and a 12.4 MP RGB sensor mounted in a 3-axis electronic gimbal [17]. Its remote controller, which serves as ground station, enables the connection to a smartphone. Depending on the objective, there are different applications to perform image and video acquisition for this UAV model. In this study, it is intended to perform an autonomous flight to acquire aerial imagery for photogrammetric processing. As such, mission planning and data acquisition was done using Pix4Dcapture (Pix4D SA, Lausanne, Switzerland) in an Android smartphone: it enables the user to define an area to survey, setting different parameters, such as flight height, camera angle, UAV speed and images' overlap.

The UAV-based imagery acquisition was conducted in 21 May 2017 at 3 PM (GMT). A single-grid flight was performed at a flight height of $100 \mathrm{~m}$ from the take-off point, covering a $255 \mathrm{~m} \times 150 \mathrm{~m}$ area. The RGB sensor was point towards a nadiral position, with a front overlap of $80 \%$ and a side overlap of $70 \%$. These parameters were chosen based on previous studies [18]. A total of 102 images were acquired in four minutes, with a ground sample distance (GSD) of approximately $4 \mathrm{~cm}$. 


\subsection{Photogrammetric Processing}

The acquired UAV-based RGB high-resolution imagery was subjected to photogrammetric processing. This step was achieved using Pix4Dmapper Pro (Pix4D SA, Lausanne, Switzerland), which can transform a set of images into point cloud data using Structure from Motion (SfM) algorithms, by identifying common tie points. Moreover, it enables to compute different data products for geodetic analysis. The processing pipeline of Pix4Dmapper Pro is divided into three main steps: (i) initial processing, which is responsible for camera position, orientation optimization, computation of tie points, and for creating a sparse point cloud; (ii) point cloud and mesh, where the sparse point cloud is used, along with the imagery, to compute a dense point cloud; and (iii) DSM, orthophoto mosaic and index, which is mostly responsible for providing orthorectified raster outcomes by point cloud interpolation, such as digital surface models (DSMs), orthophoto mosaics, digital terrain models (DTMs) and vegetation indices (VIs) [17].

Within the scope of this study the initial processing was conducted using default parameters: a high point density was selected to generate the dense point cloud, and the latter was interpolated - by means of the inverse distances weighted (IDW) method with noise filtering and a sharp surface smoothing approach - to generate the raster outcomes. The photogrammetric processing generates: (i) a orthophoto mosaic; (ii) a RGB representation of the acquired imagery; (iii) a DSM that consists in a raster with altitude information of the above surface objects; (iv) a DTM in the shape of a raster with altitude information about points that corresponds to ground (some areas where ground was not directly visible were interpolated); and (v) the selected VIs.

Computed VIs are presented in Table 1. VIs are arithmetic operations using the different spectral bands. Depending on the sensor, bands other than RGB can be used (e.g. near infrared) with different narrowness levels [4]. Both RGBVI and GRVI shown to be good when discriminating vegetation from non-vegetation areas [19, 20]. GBVI, BRVI, BGVI were included in this study due to the usage of the blue band.

Table 1. Vegetation indices used in this study.

\begin{tabular}{l|l|l}
\hline Vegetation index & Equation & Reference \\
\hline $\begin{array}{l}\text { Red Green Blue vegetation index } \\
(\text { RGBVI) }\end{array}$ & RGBVI $=\frac{\text { Green }^{2}-\text { Blue } \times \text { Red }}{\text { Green }^{2}+\text { Blue } \times \text { Red }}$ & Bendig et al. [21] \\
\hline $\begin{array}{l}\text { Green-Red vegetation index (GRVI) } \\
\begin{array}{l}\text { Green-Blue vegetation index } \\
(\text { GRVI })\end{array}\end{array}$ & GRVI $=\frac{\text { Green-Red }}{\text { Green }+ \text { Red }}$ & Tucker [22] \\
\hline $\begin{array}{l}\text { Blue/Red pigment index } \\
\text { Blue/Green pigment index }+ \text { Glue }\end{array}$ & BRVI $=\frac{\text { Blue }}{\text { Red }}$ & Zarco-Tejada et al. [23] \\
\hline & BGVI $=\frac{\text { Blue }}{\text { Green }}$ & Zarco-Tejada et al. [23] \\
\hline
\end{tabular}

To have height information from the above ground objects, a CHM was calculated. This process was achieved by subtracting the DTM to the DSM [24], as presented in 
(1). As such, altitude difference in both models is used as surface features height. QGIS raster calculator, a free and open source Geographic Information System (GIS) was used in this operation.

$$
\mathrm{CHM}=\mathrm{DSM}-\mathrm{DTM}
$$

Moreover, the orthophoto mosaic was converted to a different color space - the hue saturation and value (HSV) color space [25].

\subsection{Data Processing and Classification}

The simple linear iterative clustering (SLIC) algorithm [26] was used to segment images into multiple superpixels. By applying superpixels oversegmentation to an image, compact and uniform groups of pixels that have similar characteristics are formed [27]. The orthophoto mosaic was used in this process.

Then, a dataset was created with properties obtained from the different available data products, divided into three categories: structural, spectral and color. As a structural product, the $\mathrm{CHM}$ was used. Spectral products were composed by the five computed VIs. Color products are composed of the red, green, blue and the hue (from HSV) bands. Therefore, only the pixels from each cluster were considered for data extraction. Then, four features were extracted from each product: the mean, maximum, minimum and standard deviation. To avoid potential outliers, minimum and maximum features were estimated using the $10 \%$ lower and higher values, respectively.

Considering the knowledge from the study area, each cluster was classified in five classes: (I) trees, encompassing forest trees and trees for agronomic purposes; (ii) low vegetation, composed of grassland and seasonal agriculture plantations; (iii) shrubland, which considers the shrub communities existing in the area and some shrubs located in forest canopy gaps; (iv) bare soil, encompassing areas with little to no ground vegetation and dry vegetation; (v) and infrastructures, composed of man-made buildings and stone walls. This process was achieved in QGIS by converting the super pixel objects to a polygon shapefile.

With the segmentation done, the features extracted, and data classified, a random forest model was trained for classification purposes. Data was divided into $75 \%$ for training and $25 \%$ for classification. Feature importance was also evaluated. For accuracy assessment, different metrics were evaluated, namely: producer accuracy, user accuracy, and the overall accuracy. Producer accuracy is obtained by the percentage of how many objects on the map are correctly labeled, including errors of omission and user accuracy is obtained by the percentage of all objects that were correctly identified, encompassing errors of commission.

\section{Results and Discussion}

Some of the digital products obtained through the photogrammetric processing - CHM, RGBVI, BGVI, BRVI, GBVI, and GRVI - are presented in Fig. 2. Differences between the computed VIs and the CHM height are clearly visible. The photogrammetric 
processing was done in about $1 \mathrm{~h} 30 \mathrm{~m}$, using a workstation with two Intel® Xeon ${ }^{\circledR} \mathrm{CPU}$ E5-2680 v4, 128 GB RAM, two Nvidia Quadro M4000 and 1 TB SSD.
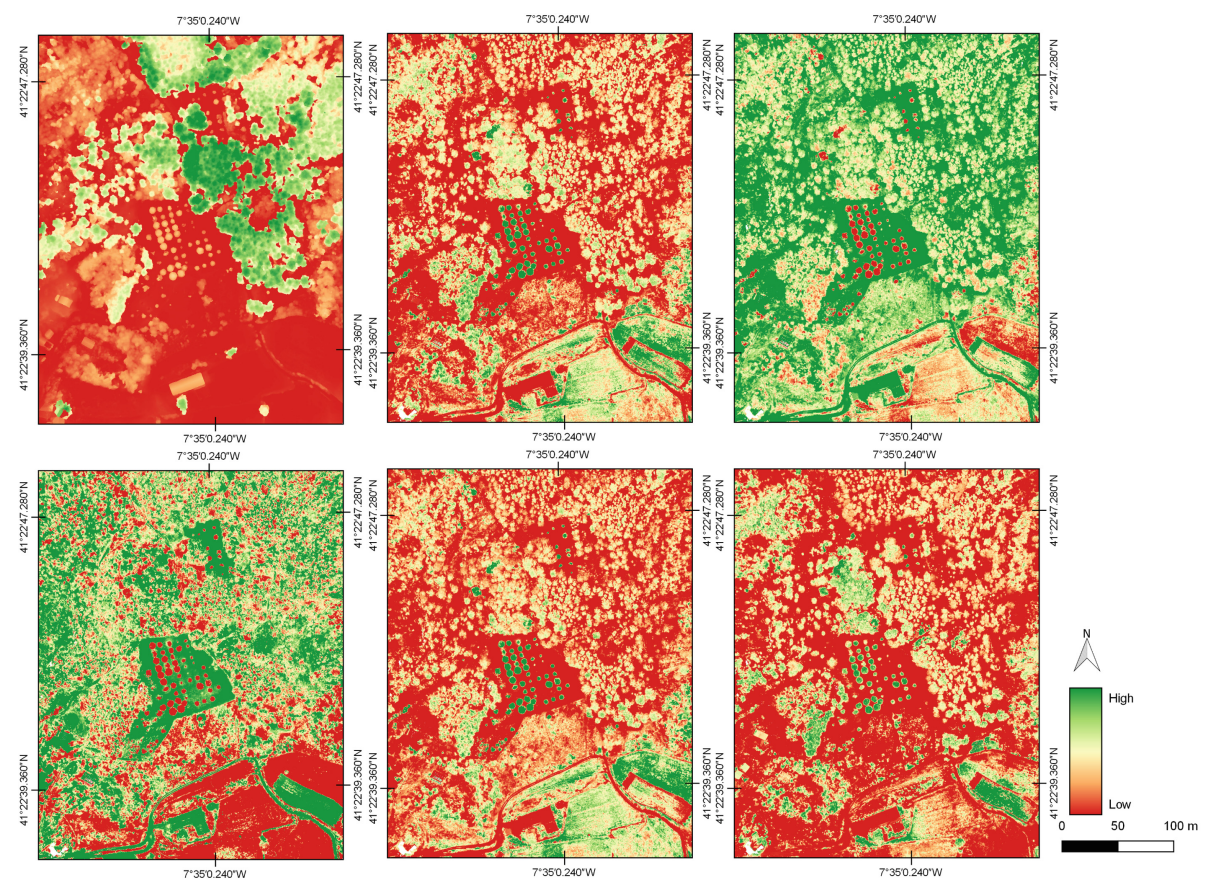

Fig. 2. Generated structural and spectral products in a color-coded representation. Top (left to right): CHM, RGBVI, and BGVI. Bottom (left to right): BRVI, GBVI, and GRVI. Coordinates in WGS84 (EPSG:4326).

The overall training accuracy, using random forest algorithm, was of $99.33 \%$. Superpixels object size varied approximately from $5 \mathrm{~m}^{2}$ to $90 \mathrm{~m}^{2}$. As for features importance (Fig. 3), spectral features (VI-based) were the most relevant (35\%), followed by color features $(34 \%)$ and color features $(31 \%)$. However, when individually analyzing each feature, the most relevant is CHM's mean value $(10.8 \%)$, followed by CHM's maximum value (10.5\%) and the blue band mean value $(7.5 \%)$. By analyzing each digital outcome individually, CHM has the highest importance (32\%). The VI with higher feature importance was the GRVI (12\%). Considering color features, both blue and red bands had the higher importance (11\% and $9 \%$, respectively). When regarding feature parameter type (i.e. mean, maximum, minimum, and standard deviation) the mean value showed the higher percentage (34\%), followed by the maximum (29\%), minimum (23\%) and, lastly, the standard deviation (14\%).

A confusion matrix with the random forest classification results is provided in Table 2. The overall accuracy was $86.4 \%$. Producer accuracy was higher for low vegetation class $(91 \%)$, followed by tree class $(90 \%)$, bare soil $(86 \%)$, shrubland $(79 \%)$ and 
infrastructure class (73\%). User accuracy was higher for low vegetation (94\%), followed by tree class $(93 \%)$, infrastructure $(89 \%)$, bare soil $(84 \%)$, and shrubland $(77 \%)$.

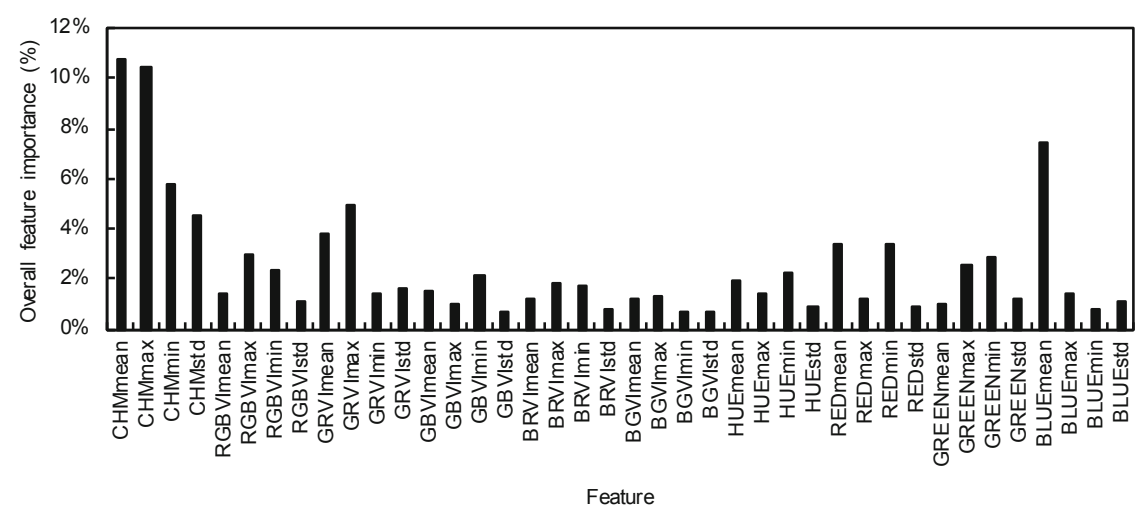

Fig. 3. Feature importance obtained from the random forest training process.

Table 2. Confusion matrix, with user accuracy, and producer accuracy for all classes, based on the number of detected superpixels.

\begin{tabular}{l|r|l|r|r|l|l|l}
\hline & Trees & $\begin{array}{l}\text { Low } \\
\text { vegetation }\end{array}$ & Shrubland & $\begin{array}{l}\text { Bare } \\
\text { soil }\end{array}$ & Infrastructure & $\begin{array}{l}\text { Producer } \\
(\%)\end{array}$ & $\begin{array}{l}\text { User } \\
(\%)\end{array}$ \\
\hline Trees & 433 & 0 & 37 & 9 & 0 & 90.40 & 92.72 \\
\hline $\begin{array}{l}\text { Low } \\
\text { vegetation }\end{array}$ & 0 & 94 & 5 & 4 & 0 & 91.26 & 94.00 \\
\hline Shrubland & 26 & 3 & 255 & 36 & 1 & 79.44 & 77.04 \\
\hline Bare soil & 8 & 2 & 33 & 269 & 0 & 86.22 & 84.33 \\
\hline Infrastructure & 0 & 1 & 1 & 1 & 8 & 72.73 & 88.89 \\
\hline
\end{tabular}

Feature importance measures shown a high value for the structural variables. Spectral VIs did not show greater importance, except for GRVI, being GBVI and BGVI the VIs with the lower importance. Among color features, blue and red bands shown the higher importance, with hue band from HSV color space not presenting a high importance. In the study of Melville et al. [11], a DSM was used, along with spectral bands driven from an hyperspectral sensor for grassland communities classification and similarly to this study, structural features had higher importance when compared with spectral features. As for feature parameter type, the mean and maximum were the most important: both presented more discrepancies between the different classes. As for minimum and standard deviation features, they shown lower importance. This can be related to the similarities of these features in all classes. For example, a superpixel object with a tree and a small portion of soil can present the same minimum value as an object from the soil class. 
Regarding classification results (Table 2), some confusion between tree and shrubland classes was observed. This can be explained by the height similarity between some younger pine trees and shrubs, as well as the difficulty of photogrammetric techniques in penetrating the canopy [18], which results in wrong CHM estimates. As such, situations where shrublands are located in canopy gaps can be classified as being a tree. Furthermore, some soil objects were classified as shrub. These were related with objects located in shadowed areas. As for infrastructure results, these can be explained with the low number of objects representing this class (it was the less represented class), showing some imbalance in the dataset. The final classification image and its reference are presented in Fig. 4.

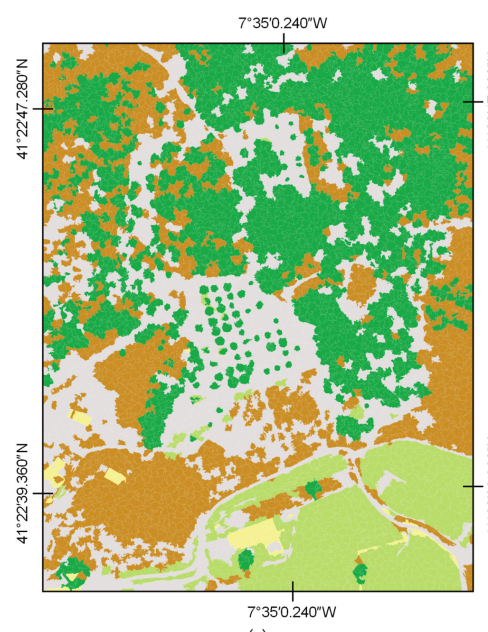

(a)

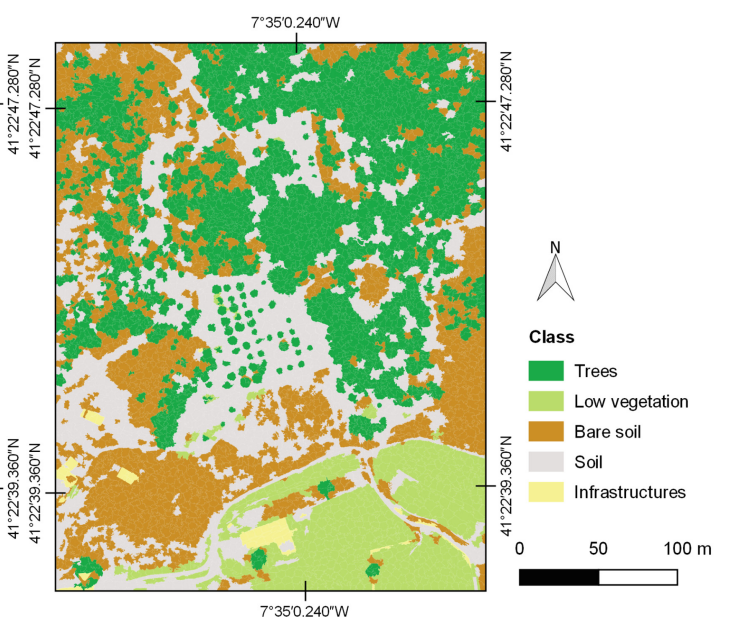

(b)

Fig. 4. Ground-truth classification (a) and random forest classification (b) of the study area. Coordinates in WGS84 (EPSG:4326).

\section{Conclusions}

This paper demonstrates the usefulness of random forest algorithm for classification of photogrammetric products obtained from UAV-based RGB imagery in an agrosilvopastoral system. Results indicated that UAV-based RGB imagery provides enough detail to classify different vegetation and non-vegetation objects. Moreover, encouraging accuracies were obtained in the random forest classification of the evaluated classes, being this a suitable approach for the characterization of agrosilvopastoral systems. The dataset used in this study shown to be effective for this purpose. Still, some concerns towards feature type selection must be considered in future studies.

Future developments should rely in exploring data acquisition in different flight heights, to obtain the best compromise towards data acquisition time and results accuracy. Other aspect is to explore other UAV-based imagery type, such as multispectral and thermal infrared imagery. Multispectral sensors can acquire data from 
other near infrared regions of the electromagnetic spectrum, which combined with temperature, may improve the classification results.

Acknowledgments. This work is financed by the ERDF - European Regional Development Fund through the Operational Programme for Competitiveness and Internationalisation COMPETE 2020 Programme within project «POCI-01-0145-FEDER-006961» and by National Funds through the FCT - Fundação para a Ciência e a Tecnologia (Portuguese Foundation for Science and Technology) as part of project UID/EEA/50014/2013. The research activities of Luís Pádua were funded by the Portuguese Foundation for Science and Technology (SFRH/ $\mathrm{BD} / 139702 / 2018)$.

\section{References}

1. Torresan, C., et al.: Forestry applications of UAVs in Europe: a review. Int. J. Remote Sens. 38, 2427-2447 (2017). https://doi.org/10.1080/01431161.2016.1252477

2. Castaldi, F., Pelosi, F., Pascucci, S., Casa, R.: Assessing the potential of images from unmanned aerial vehicles (UAV) to support herbicide patch spraying in maize. Precision Agric. 18, 76-94 (2017). https://doi.org/10.1007/s11119-016-9468-3

3. von Bueren, S.K., Burkart, A., Hueni, A., Rascher, U., Tuohy, M.P., Yule, I.J.: Deploying four optical UAV-based sensors over grassland: challenges and limitations. Biogeosciences 12, 163-175 (2015). https://doi.org/10.5194/bg-12-163-2015

4. Pádua, L., et al.: UAS, sensors, and data processing in agroforestry: a review towards practical applications. Int. J. Remote Sens. 38, 2349-2391 (2017). https://doi.org/10.1080/ 01431161.2017.1297548

5. Matese, A., et al.: Intercomparison of UAV, aircraft and satellite remote sensing platforms for precision viticulture. Remote Sens. 7, 2971-2990 (2015). https://doi.org/10.3390/ rs70302971

6. Shakhatreh, H., et al.: Unmanned aerial vehicles: a survey on civil applications and key research challenges. arXiv:1805.00881 [cs] (2018)

7. Belgiu, M., Drăguț, L.: Random forest in remote sensing: a review of applications and future directions. ISPRS J. Photogram. Remote Sens. 114, 24-31 (2016). https://doi.org/10.1016/j. isprsjprs.2016.01.011

8. Feng, Q., Liu, J., Gong, J.: UAV remote sensing for urban vegetation mapping using random forest and texture analysis. Remote Sens. 7, 1074-1094 (2015). https://doi.org/10.3390/ rs70101074

9. Akar, Ö.: The rotation forest algorithm and object-based classification method for land use mapping through UAV images. Geocarto Int. 33(5). https://www.tandfonline.com/doi/abs/ 10.1080/10106049.2016.1277273

10. Ma, L., et al.: Evaluation of feature selection methods for object-based land cover mapping of unmanned aerial vehicle imagery using random forest and support vector machine classifiers. IJGI 6, 51 (2017). https://doi.org/10.3390/ijgi6020051

11. Melville, B., Lucieer, A., Aryal, J.: Classification of lowland native grassland communities using hyperspectral unmanned aircraft system (UAS) imagery in the tasmanian midlands. Drones 3, 5 (2019). https://doi.org/10.3390/drones3010005

12. Nevalainen, O., et al.: Individual tree detection and classification with UAV-based photogrammetric point clouds and hyperspectral imaging. Remote Sens. 9, 185 (2017). https://doi.org/10.3390/rs9030185 
13. Michez, A., Piégay, H., Lisein, J., Claessens, H., Lejeune, P.: Classification of riparian forest species and health condition using multi-temporal and hyperspatial imagery from unmanned aerial system. Environ. Monit. Assess. 188, 146 (2016). https://doi.org/10.1007/s10661-0154996-2

14. Goodbody, T.R.H., Coops, N.C., Hermosilla, T., Tompalski, P., Crawford, P.: Assessing the status of forest regeneration using digital aerial photogrammetry and unmanned aerial systems. Int. J. Remote Sens. 39, 5246-5264 (2018). https://doi.org/10.1080/01431161. 2017.1402387

15. Russo, R.O.: Agrosilvopastoral systems: a practical approach toward sustainable agriculture. J. Sustain. Agric. 7, 5-16 (1996). https://doi.org/10.1300/J064v07n04_03

16. Nair, P.K.R.: Classification of agroforestry systems. Agroforest Syst. 3, 97-128 (1985). https://doi.org/10.1007/BF00122638

17. Pádua, L., et al.: UAS-based imagery and photogrammetric processing for tree height and crown diameter extraction. In: Proceedings of the International Conference on Geoinformatics and Data Analysis, pp. 87-91. ACM, New York (2018). https://doi.org/10.1145/ 3220228.3220241

18. Dandois, J.P., Olano, M., Ellis, E.C.: Optimal altitude, overlap, and weather conditions for computer vision UAV estimates of forest structure. Remote Sens. 7, 13895-13920 (2015). https://doi.org/10.3390/rs71013895

19. Motohka, T., Nasahara, K.N., Oguma, H., Tsuchida, S.: Applicability of green-red vegetation index for remote sensing of vegetation phenology. Remote Sens. 2, 2369-2387 (2010). https://doi.org/10.3390/rs2102369

20. Marques, P., et al.: UAV-based automatic detection and monitoring of chestnut trees. Remote Sens. 11, 855 (2019). https://doi.org/10.3390/rs11070855

21. Bendig, J., et al.: Combining UAV-based plant height from crop surface models, visible, and near infrared vegetation indices for biomass monitoring in barley. Int. J. Appl. Earth Obs. Geoinf. 39, 79-87 (2015). https://doi.org/10.1016/j.jag.2015.02.012

22. Tucker, C.J.: Red and photographic infrared linear combinations for monitoring vegetation. Remote Sens. Environ. 8, 127-150 (1979). https://doi.org/10.1016/0034-4257(79)90013-0

23. Zarco-Tejada, P.J., et al.: Assessing vineyard condition with hyperspectral indices: leaf and canopy reflectance simulation in a row-structured discontinuous canopy. Remote Sens. Environ. 99, 271-287 (2005). https://doi.org/10.1016/j.rse.2005.09.002

24. Popescu, S.C.: Estimating biomass of individual pine trees using airborne lidar. Biomass Bioenerg. 31, 646-655 (2007). https://doi.org/10.1016/j.biombioe.2007.06.022

25. Smith, A.R.: Color gamut transform pairs. In: Proceedings of the 5th Annual Conference on Computer Graphics and Interactive Techniques - SIGGRAPH 1978, pp. 12-19. ACM Press (1978). https://doi.org/10.1145/800248.807361

26. Achanta, R., Shaji, A., Smith, K., Lucchi, A., Fua, P., Süsstrunk, S.: SLIC superpixels compared to state-of-the-art superpixel methods. IEEE Trans. Pattern Anal. Mach. Intell. 34, 2274-2282 (2012). https://doi.org/10.1109/TPAMI.2012.120

27. Crommelinck, S., Bennett, R., Gerke, M., Koeva, M.N., Yang, M.Y., Vosselman, G.: SLIC superpixels for object delineation from UAV data. ISPRS Ann. Photogramm. Remote Sens. Spatial Inf. Sci. 4, 9-16 (2017). https://doi.org/10.5194/isprs-annals-IV-2-W3-9-2017 